\title{
A creatina como suplemento ergogênico para atletas
}

\section{Creatine as an ergogenic supplement for athletes}

José PERALTA ${ }^{1}$

Olga Maria Silverio AMANCIO²

\section{R E S U M O}

A creatina vem sendo muito pesquisada devido ao seu potencial efeito no rendimento físico de atletas envolvidos em exercícios de alta intensidade e curta duração, intermitentes e com curtos períodos de recuperação. A creatina fosforilada é uma reserva de energia nas células musculares. Durante um exercício intenso, a sua quebra libera energia é usada para regenerar o trifosfato de adenosina. Aproximadamente $95 \%$ do pool de creatina encontra-se na musculatura esquelética e sua regeneração após o exercício é um processo dependente de oxigênio. Estudos mostram que a suplementação com este composto pode aumentar o pool orgânico em 10 a $20 \%$, e este percentual é maior em atletas vegetarianos (até $60 \%$ ). Ainda existe controvérsia com relação aos benefícios e riscos da suplementação com esta substância. Este estudo revisa alguns dos aspectos relacionados com o metabolismo da creatina e seu uso como substância ergogênica na prática desportiva.

Termos de indexação: creatina, suplementação alimentar, metabolismo, exercício.

\section{A B S T R A C T}

Several researches on creatine have been done due to its potential effects on the physical performance of athletes involved in high intensity, short duration and intermittent exercises with short periods of recovery. Phosphorylated creatine is an energy reserve in the muscle cells. During an intense exercise, its breakdown liberates energy used to regenerate adenosine triphosphate. Approximately $95 \%$ of the creatine pool is found in the skeletal muscle, and the regenerating process after exercise is oxygen dependent. Studies show that supplementation with this compound may procedure an increase of $10 \%$ to $20 \%$ in the organic pool, and this percentage is higher in vegetarian athletes (up to 60\%). There is still controversy regarding the benefits and risks of supplementation with this substance. This paper reviews some aspects related to the creatine metabolism and its use as an ergogenic substance in sports practice.

Index terms: creatine, supplementary feeding, metabolism, exercise.

\footnotetext{
1 Curso de Pós-graduação Ciências Aplicadas à Pediatria, Departamento de Pediatria, Universidade Federal de São Paulo/Escola Paulista de Medicina. Rua Botucatu, 703, 04023-062, São Paulo, SP, Brasil. Correspondência para/Correspondence to: J. PERALTA.

2 Departamento de Pediatria, Universidade Federal de São Paulo/Escola Paulista de Medicina.
} 


\section{N T R O D U Ç Ã O}

A creatina já era conhecida desde o século passado (Greenhaff, 1995); porém, sua função no metabolismo muscular e no desempenho físico tornou-se motivo de interesse nos anos recentes.

Na célula muscular, a creatina em sua forma fosforilada, creatina-fosfato (CP), constitui uma reserva de energia para a rápida regeneração do trifosfato de adenosina (ATP), em exercícios de alta intensidade e curta duração, como por exemplo, durante um sprint de $100 \mathrm{~m}$ rasos ou em uma seqüência de levantamento de peso em um treino de halterofilismo.

A creatina orgânica tem duas fontes, a síntese pelo próprio organismo, a partir de 3 aminoácidos; e a ingestão de alimentos, especificamente das carnes (Redondo et al., 1996). O pool orgânico desta substância encontra-se localizado quase na sua totalidade (95\%) na musculatura esquelética e sua regeneração após um exercício intenso é um processo dependente da via oxidativa (Mayes, 1996).

A maioria dos estudos de suplementação com creatina tem mostrado a possibilidade de aumentar o pool orgânico deste composto em 10 a $20 \%$, embora alguns estudos tenham evidenciado acréscimo de até $50 \%$ em seus níveis totais, após a suplementação em indivíduos não vegetarianos (Burke \& Berning, 1996). Estes autores relatam que em atletas vegetarianos 0 aumento chega em torno dos $60 \%$. A ampliação da reserva de energia no músculo através da creatina tem permitido aprimorar o desempenho físico de atletas (Volek et al., 1997; Ziegenfuss et al., 1997). Contudo, nem todas as pesquisas têm mostrado efeitos significativos após a suplementação com este composto (Dawson et al., 1995; Terrillion et al., 1997).

São considerados recursos ergogênicos as substâncias (entre elas a creatina), os processos, ou os procedimentos que podem, ou são percebidos como sendo capazes de melhorar o desempenho esportivo (Williams, 1998). O consumo de alguns destes recursos ergogênicos pode ter resultado positivo para as provas de dopagem, portanto são vetados pelo Comitê Olímpico Internacional (COI). A creatina está fora da lista de substâncias proibidas pelo $\mathrm{COI}$, por isso seu consumo não é considerado como doping.

No meio esportivo, esta substância foi popularizada nos Jogos Olímpicos de 1992, em Barcelona, quando o corredor britânico Linford Christie, ganhador da medalha de ouro nos $100 \mathrm{~m}$ rasos, creditou sua vitória ao consumo da creatina.

Ainda não há consenso sobre os efeitos colaterais resultantes do consumo de creatina por tempo prolongado. São necessárias campanhas de informação e conscientização sobre a real necessidade de consumo de suplementos para a população fisicamente ativa que não pratica exercícios físicos profissionalmente.

Este trabalho tem por objetivo revisar alguns aspectos relacionados ao metabolismo da creatina e ao seu uso como uma substância ergogênica na prática desportiva.

Durante uma prova de corrida, a cada passo dado por um competidor, uma quantidade próxima de $10^{19}$ moléculas de ATP são convertidas em difosfato de adenosina ADP, com a correspondente transferência de energia para o trabalho muscular; de certa forma, isto significaria que um maratonista poderia gastar o equivalente a $75 \mathrm{~kg}$ de ATP durante a corrida (Newsholme et al., 1994). Como o atleta não poderia levar esta quantidade de ATP, este fato é resolvido pela rápida regeneração da molécula a partir do ADP e do fosfato inorgânico e a energia fornecida por outro composto de reserva: pela creatina fosfato $(\mathrm{CP})$.

\section{Fornecimento anaeróbio de energia: sistema ATP - CP}

Durante os primeiros segundos de um exercício intenso, a concentração muscular de ATP é mantida em nível mais ou menos constante. $\mathrm{O}$ 
ATP utilizado é rapidamente reposto a partir da quebra da CP. Assim, os níveis de CP diminuem rapidamente à medida em que este composto é usado para regenerar o ATP.

Quando o exercício físico é levado até a exaustão, tanto as concentrações musculares de ATP quanto as de CP ficam diminuídas, tornando-as indisponíveis para o fornecimento energético de forma eficiente para a continuação do trabalho muscular.

Embora haja evidências de que a concentração muscular de ATP possa cair a quase zero (estudos com cavalos), esta situação não tem sido mostrada em humanos, nos quais o conteúdo total de ATP muscular pode cair em aproximadamente $50 \%$ no ponto de fadiga, durante um exercício de alta intensidade (Spriet, 1995).

A concentração de ATP na maioria dos tecidos é baixa, cerca de 3 a $8 \mathrm{mmol} / \mathrm{kg}$. A concentração de CP no músculo é 4 a 5 vezes a do ATP (em média $18 \mathrm{mmol} / \mathrm{kg}$ músculo), representando uma quantidade aproximada de $120 \mathrm{~g}$ de creatina total em um indivíduo adulto de 70 kg (Guerrero-Ontiveros \& Wallimann, 1998). Esta reserva, embora limitada, é suficiente para atuar como um tampão temporário de ATP, até outros processos regeneradores do ATP atingirem sua máxima velocidade (Houston, 1995).

O ATP e a CP, juntos, podem proporcionar energia para os músculos por um tempo de aproximadamente 3 a 12 segundos (Burke \& Berning, 1996).

Durante o processo de contração muscular, o ATP utilizado para a geração de energia é quebrado pela enzima ATPase em uma reação muito rápida. O ADP resultante é prontamente regenerado, a partir da CP, pela ação de outra enzima, a creatina-quinase (CK). Esta reação, livremente reversível, está invertida durante o repouso, no sentido de favorecer a regeneração da $C P$, usando a energia disponível através do processo oxidativo, que ocorre dentro da mitocôndria (Houston, 1995), sugerindo-se que a taxa inicial de recuperação da CP seria proporcional à taxa mitocondrial de consumo de oxigênio (Thompson et al., 1995).

\section{Metabolismo da creatina}

A creatina é um aminoácido, ácido metil guanidina - acético (Kreider, 1998), o qual se encontra presente tanto nos alimentos quanto no organismo humano, devido à síntese endógena.

Nos alimentos, a creatina é encontrada em maior quantidade nas carnes (todos os tipos): bacalhau - 3,0; linguado - 2,0; salmão - 4,5; atum - 4,0; e carne bovina - 4,5 g/kg (Balsom et al., 1994). Encontra-se também em outros alimentos, porém, em quantidades muito pequenas.

Quando sintetizado no homem, este composto nitrogenado inicia seu ciclo de formação no rim, em uma reação envolvendo dois aminoácidos: arginina e glicina. Esta operação, catalisada pela enzima transaminidase, ocorre apenas neste órgão. Posteriormente, a creatina completa sua síntese pela adição de um grupo metil fornecido a partir da metionina (S-adenosilmetionina). Esta reação ocorre no fígado (Rodwell, 1996). A creatina assim formada, fora do músculo, é então distribuída para os diversos tecidos do organismo através do sangue.

A concentração plasmática de creatina é muito pequena, entre 50 e $100 \mu \mathrm{mol} / \mathrm{L}$ (Balsom et al., 1994). Na urina encontram-se apenas traços deste composto.

O principal destino final da creatina sintetizada é o tecido muscular esquelético, o qual detém aproximadamente 95\% do pool orgânico; os $5 \%$ restantes distribuem-se entre órgãos como o coração, cérebro, retina e testículos (Balsom et al., 1994).

A captação da creatina pelas células musculares é um processo saturável que ocorre ativamente contra um gradiente de concentração (transportador sódio-dependente), possivelmente 
envolvendo a interação da creatina com sítios específicos da membrana que reconhecem parte da molécula da creatina (Greenhaff, 1997). Alguns possíveis mecanismos reguladores do armazenamento intracelular de creatina têm sido sugeridos: em um deles, na síntese endógena no rim e fígado, a concentração de creatina influenciaria um controle de feedback negativo na enzima que catalisa a $1^{\text {a }}$ reação de síntese (transferase); em outro, a homeostase da creatina seria regulada pelo controle da expressão e atividade da proteína transportadora de creatina, podendo ser afetada por diversos análogos estruturais desta substância ou inibidores metabólicos (Guerrero-Ontiveros \& Wallimann, 1998).

Diariamente, um indivíduo adulto, com uma dieta habitual variada (mista), ingere aproximadamente $1 \mathrm{~g}$ de creatina, e uma quantidade similar é produzida pelo fígado para atingir as necessidades diárias. Este total (cerca de $2 \mathrm{~g}$ ), equivale aproximadamente à creatina reciclada diariamente pelo organismo (Greenhaff, 1995).

No músculo esquelético existe um equilíbrio reversível entre a creatina e a creatina-fosfato: na condição de repouso, aproximadamente dois terços do conteúdo de creatina está na forma fosforilada (CP) e o restante fica na forma livre (Balsom et al., 1994). Como anteriormente referido, a fosforilação da creatina é um processo dependente de oxigênio, isto é, da fosforilação oxidativa (Mayes, 1996).

A taxa de degradação da CP tem se mostrado maior nas fibras musculares do tipo II (contração rápida) em relação às do tipo I (contração lenta), e a disponibilidade de CP como um substrato energético nas fibras de contração rápida é considerada o possível fator limitante para a manutenção da força muscular durante um exercício de alta intensidade (Balsom et al., 1994; Greenhaff et al., 1994).

Por outro lado, Gariod et al. (1994) mostraram que a taxa de ressíntese da CP nas fibras musculares do tipo I é mais rápida do que nas do tipo II, devido, provavelmente, ao maior potencial aeróbio das fibras de contração lenta; portanto, este é um processo dependente de oxigênio.

Após um exercício de alta intensidade, aproximadamente metade da concentração inicial de $C P$ é regenerada no primeiro minuto de recuperação; a ressíntese total da CP ficaria completada após aproximadamente 5 minutos (Soderlund \& Hultman, 1991).

Embora a CP não seja considerada como uma fonte energética primária durante os exercícios submáximos, tem sido relatada uma relação inversa entre a intensidade do exercício e o nível de CP nos músculos exercitados. Em indivíduos submetidos a exercícios de bicicleta em uma intensidade entre 60 e $70 \%$ do $\mathrm{VO}_{2}$ max. durante 80 minutos, tem se demonstrado uma diminuição dos níveis de CP para aproximadamente $40 \%$ dos valores iniciais (Broberg \& Sahlin, 1989). Assim, parece que estas quantidades diminuem até mesmo durante o exercício submáximo, mas os estoques musculares não são consumidos no mesmo grau como em um exercício de alta intensidade. Segundo Willmore \& Costill (1994), os níveis de CP podem ser reduzidos em 10 - 15\% do conteúdo inicial, após 10 - 12 segundos de exercício intenso.

A creatina na forma fosforilada, como reserva de fosfatos de alta energia, não permite unicamente a manutenção dos níveis de ATP intracelular em condições de trabalho muscular, mas também, na condição de repouso (recuperação), atua no músculo como transportador de grupos fosfato de alta energia da mitocôndria para o citoplasma (Mayes, 1996). Este mecanismo, na célula muscular esquelética e cardíaca, permite o rápido deslocamento de fosfatos de alta energia da matriz mitocondrial, onde são produzidos a partir da fosforilação oxidativa, até o citosol.

A CP também é utilizada durante o trabalho anaeróbio (láctico) intermediário - 15 segundos até alguns minutos, quando sua quebra 
vai ajudar a tamponar o meio ácido intracelular, causado pelo acúmulo de lactato. Maior quantidade de CP na célula muscular significa uma maior capacidade de tamponamento, e assim, um maior tempo de resistência à fadiga (Soderlund et al., 1994).

O ciclo da creatina finaliza quando é convertida em creatinina, por uma reação contínua e irreversível de desidratação (não enzimática), sendo excretada pela urina. A quantidade de creatinina reciclada e eliminada é constante de um dia para outro e é produzida em proporção à massa muscular de um indivíduo (Devlin, 1992). Segundo Forbes (1991), em indivíduos que consomem uma dieta mista (sem consumo de suplementos de creatina), a relação entre massa muscular (MM) e excreção de creatinina ( $\mathrm{Cr}$ ) é a seguinte: $M M(k g)=14,3 \mathrm{Cr}(\mathrm{g} / \mathrm{d})+3,6$.

\section{Fatores relacionados ao armazenamento de creatina no músculo}

Ainda não é claro se existem diferenças devido ao sexo para a concentração de CP armazenada no músculo. Em um estudo realizado por Soderlund (1992), com 20 homens e 25 mulheres, não foi observada nenhuma diferença estatisticamente significante entre as concentrações médias de creatina, segundo o sexo $(127,7 \pm 2,1$ e $131,4 \pm 2,4 \mathrm{mmol} / \mathrm{kg}$ de músculo seco, respectivamente).

Em relação à idade, McCully \& Posner (1992) relataram que a taxa de ressíntese da CP após um exercício tende a diminuir com o tempo, aproximadamente $8 \%$ a cada 10 anos após os 30 anos de idade.

A redução do tamanho da massa muscular, a diminuição do diâmetro das fibras do tipo II e a diminuição da atividade enzimática mitocondrial e do metabolismo dos fosfatos de alta energia são algumas das alterações relacionadas com a idade que se associam com o declínio da força muscular e da capacidade de resistência física, o qual ocorre com o envelhecimento (Smith et al., 1998).

Moller et al. (1980) não encontraram nenhuma diferença na quantidade total de creatina entre indivíduos idosos (entre 52 e 79 anos) e jovens (entre 18 e 36 anos). Apesar deste achado, observou-se na população idosa uma menor quantidade de CP e maior de creatina livre, quando comparada com a população jovem. Os autores sugeriram ser este resultado uma conseqüência da inatividade física dos indivíduos mais velhos. A hipótese foi reafirmada com uma pesquisa posterior, na qual encontrou-se uma inversão das proporções da CP e da creatina livre, sem ter havido qualquer mudança na concentração total de creatina no músculo, quando um programa de treinamento foi introduzido (Moller \& Brandt, 1981).

Smith et al. (1998) realizaram um estudo com um grupo de 5 indivíduos jovens (idade média de 30 anos) e 4 adultos (média de 58 anos), os quais foram suplementados com creatina $(0,3 \mathrm{~g} / \mathrm{kg} / \mathrm{d})$ por 5 dias. Antes da suplementação, os jovens tinham uma concentração de $C P$ muscular e taxa inicial de ressíntese significativamente maior do que os adultos. Após a suplementação, a concentração de CP em repouso aumentou em 15\% nos jovens e 30\% nos adultos, resultando em ambos os grupos em uma taxa inicial similar de ressíntese de CP.

A determinação da composição dos tipos de fibras musculares não varia somente de um músculo para outro, mas também entre indivíduos. Este é o motivo de alguns serem melhores corredores de velocidade e outros melhores corredores de endurance (Newsholme et al., 1994). Segundo técnicas de separação de fibras musculares dos tipos I e II, a partir de amostras de tecido muscular congelado e seco, as fibras do tipo II (contração rápida), em repouso, tiveram maior concentração muscular de CP do que as fibras do tipo I (contração lenta) (Soderlund et al., 1992). Confirmando estes resultados, Edström et al. (1982) demonstraram concentração de CP significamente menor no músculo sóleo 
em humanos (contendo aproximadamente 65\% de fibras do tipo I) em relação ao músculo vasto lateral (com aproximadamente $41 \%$ das fibras do tipo I).

\section{Suplementação com creatina}

A creatina como suplemento parece não aumentar a concentração de ATP muscular de repouso, mas parece ajudar a manter os níveis de ATP durante um esforço físico máximo (Greenhaff et al., 1993). A suplementação com este composto aumenta o pool de creatina corporal, o que potencialmente facilitaria a geração de maior quantidade de CP. O efeito ergogênico pode ser específico para certos tipos de esforço físico, como por exemplo: exercícios repetitivos (intermitentes), de alta intensidade, curta duração e com períodos de recuperação muito curtos (American College..., 1999). É possível ainda a suplementação com creatina permitir ao atleta se engajar em um treino físico mais intenso, o qual eventualmente poderia se traduzir em uma melhora do desempenho físico (Kreider, 1998; Williams, 1998).

A suplementação para atletas é feita na forma de creatina monohidratada, um pó branco solúvel em água. A quantidade armazenada de creatina durante a suplementação é muito variável entre indivíduos; estas variações sugerem que a captação desta substância é dependente de diferentes fatores, incluindo diferenças na composição da dieta, conteúdo muscular inicial deste composto, sexo, composição de fibras musculares (Guerreiro-Ontiveros \& Wallimann, 1998).

Os atletas vegetarianos quem mais se beneficiam com a suplementação com creatina. Suas dietas não contêm fontes deste composto, por isso, apresentam baixos níveis deste elemento no organismo. O consumo de suplementos desta substância tem mostrado possibilidade de aumento na concentração de creatina muscular de aproximadamente $60 \%$, quando comparado com outro grupo alimentado com dieta mista (10 - 20\%) (Burke \& Berning, 1996).

O consumo de creatina junto com glicose, cerca de $100 \mathrm{~g}$, aumenta o conteúdo muscular deste composto em aproximadamente 10\%, conforme exposto por Green et al. (1996). Há uma elevação da captação de creatina pela fibra muscular, e, conseqüentemente, sua ingestão com este carboidrato simples pode aumentar o efeito ergogênico. O processo parece ser mediado pela insulina, a qual estimularia a enzima ATPase da bomba de $\mathrm{Na}^{+} / \mathrm{K}^{+}$, que por sua vez promoveria um transporte simultâneo de $\mathrm{Na}^{+} /$Creatina (duas moléculas de sódio para cada uma de creatina) para manter ou restaurar o gradiente normal de $\mathrm{Na}^{+}$e o potencial de membrana (Odoom et al., 1996).

Após a ingestão de $5 \mathrm{~g}$ de creatina, o nível plasmático aumenta de uma faixa entre 50 e $100 \mu \mathrm{mol} / \mathrm{L}$ para mais de $500 \mu \mathrm{mol} / \mathrm{L}$, uma hora após o seu consumo (Harris et al., 1992). Doses diárias de $20 \mathrm{~g}$ (divididas em 4 ou 5 vezes), por um período de 5 a 7 dias, geralmente elevam o conteúdo total desta substância no músculo em cerca de 10 a 20\% (Grindstaff et al., 1997; Kreider, 1998). Contudo, recentemente tem sido evidenciadas quantidades menores, $3 \mathrm{~g} / \mathrm{dia}$ por 30 dias, apresentando o mesmo efeito (American College..., 1999). Assim, altas doses (20 g/dia) seriam desnecessárias para aumentar o conteúdo deste composto no músculo. Aproximadamente um terço da creatina extra que ingressa no músculo é fosforilada (Balsom et al., 1995; Burke \& Berning, 1996) e o restante predomina na forma livre (Vandenberghe et al., 1997).

Vandenberghe et al. (1996) lançaram a hipótese de o consumo oral de creatina combinada com algum estímulo adrenérgico - por exemplo, com o consumo de cafeína - poder facilitar a acumulação muscular de creatina. A cafeína tem mostrado estimular diretamente a atividade da ATPase da bomba de $\mathrm{Na}^{+} / \mathrm{K}^{+}$muscular e aumentar os níveis plasmáticos de epinefrina, estímulos diretos para a atividade do referido mecanismo. Surpreendentemente, resultados 
iniciais indicaram que a cafeína não melhorava a eficiência da suplementação oral de creatina, não aumentava os níveis musculares de $C P$, nem aperfeiçoava o desempenho físico. A cafeína teria suprimido completamente o efeito ergogênico da suplementação de creatina.

Por um lado, são diversas as pesquisas relevando melhora do desempenho físico e/ou mudanças na composição corporal em atletas em função da suplementação com creatina (Balsom et al., 1995; Vandenberghe et al., 1996; Grindstaff et al., 1997; Vandenberghe et al., 1997; Volek et al., 1997; Ziegenfuss et al., 1997). Por outro lado, estudos bem controlados não têm evidenciado alterações significativas na performance, após esta suplementação (Dawson et al., 1995; Redondo et al., 1996; Terrillion et al., 1997).

Conseqüentemente, embora possa ser evidenciada alguma vantagem decorrente da suplementação com este composto, nem todo indivíduo que consome tal substância será necessariamente beneficiado com um melhor desempenho desportivo.

Em uma extensa revisão de estudos a este respeito, Kreider (1998) relata resultados menos efetivos da suplementação com creatina nas seguintes situações: quando foi consumida em quantidade inferior a $20 \mathrm{~g} / \mathrm{d}$ e por um período inferior 5 dias; quando foi consumida em doses baixas (2-3 g/d), sem o período de carga inicial (dose alta); em estudos com um número limitado de indivíduos; e quando o exercício máximo (sprint) foi realizado com períodos muito curtos ou muito longos de recuperação entre os sprints.

Mulheres destreinadas que consumiram creatina $(20 \mathrm{~g} / \mathrm{d})$ por 4 dias, seguido por um consumo de $5 \mathrm{~g}$ diários por um período de 66 dias, associado a um treinamento de força, tiveram um ganho significativamente maior de massa magra $(1,0 \mathrm{~kg})$, quando comparado com o grupo-controle, segundo Vandenberghe et al. (1997).

Williams (1998) refere que apesar das pesquisas terem mostrado um aumento entre 0,9 e 2,2 kg de ganho de massa corporal após uma semana de suplementação com creatina, este acréscimo, como é de se esperar, em função do curto período de tempo, não é de massa muscular.

Duas teorias prevalecem para tentar explicar os efeitos da suplementação com a creatina: a primeira supõe que a suplementação com creatina promoveria retenção de água, provavelmente ligada a esta substância, e a diminuição da produção de urina associada à esta complementação, encontrada em alguns estudos, constituiria um marcador indireto de retenção de fluídos no corpo; a segunda presume que a suplementação realmente promoveria um aumento da síntese de proteína. Portanto, são necessários mais estudos para se ter certeza sobre a contribuição de cada um dos processos através dos quais se obteve ganho de peso (Kreider, 1998; Williams, 1998).

A suplementação com creatina não tem sido comprovada como eficaz para o aumento do desempenho físico em esportes de endurance (American College..., 1999).

\section{Riscos e desvantagens da suplementação}

As informações sobre os efeitos colaterais da suplementação com creatina provêm principalmente de comunicações anedóticas, sem um fundamento científico sólido; portanto, qualquer discussão sobre possíveis efeitos negativos da suplementação merece ser analisada com cuidado.

Kreider (1998) resume e analisa alguns fatos encontrados em publicações destinadas para leigos: a suplementação pode promover tensão muscular; quando consumida durante treinos, em clima quente, pode ocasionar câimbras musculares, e consumo de creatina aumenta o risco de problemas da função renal e de distúrbios gastrintestinais.

Segundo o mesmo autor, em relação ao primeiro ponto, pode-se dizer que o ganho de força e massa corporal (decorrente principalmente 
do treinamento) origina um estresse adicional sobre ossos e ligamentos, embora, nenhum estudo tenha documentado um aumento da incidência de lesões decorrente da suplementação com creatina, mesmo em atletas com períodos de intenso treinamento.

Em relação ao segundo ponto, a possibilidade de ocorrer câimbras pelo consumo de creatina tem sido atribuída a mudanças nas concentrações de água e sais minerais nas fibras musculares. Porém, nenhum estudo tem evidenciado que a suplementação com este composto possa ocasionar câimbras, desidratação ou mudanças nas concentrações intramusculares de eletrólitos. Estas situações podem estar mais relacionadas com a fadiga muscular e desidratação resultante do treinamento em clima quente. O organismo tem uma capacidade muito grande para eliminar uma quantidade extra de creatina (da suplementação), sem nenhum tipo de dificuldade (Poortmans et al., 1997). Pode ser eliminada na forma de creatina ou creatinina.

Quanto ao terceiro ponto, não foram encontrados estudos mostrando dano clinicamente significativo da função hepática ou renal causada pela suplementação oral com creatina (American College..., 1999).

A supressão da síntese endógena de creatina (mecanismo de feedback) pelo consumo oral deste composto é conhecida há algum tempo (Walker, 1979), mas esta situação pode ser revertida quando a suplementação é suspensa.

Pesquisas têm indicado a necessidade de aproximadamente 4 semanas, após a interrupção da ingestão de creatina, para que o conteúdo muscular desta substância e CP de voltem aos valores normais (Hultman et al., 1996; Vandenberghe et al., 1997), entretanto, ainda não é claro se o conteúdo muscular destes compostos cai abaixo dos níveis normais (basais) em um período posterior.

Em ratos, a suplementação crônica de creatina suprime a expressão da proteína transportadora deste elemento. Este resultado pode ser extrapolado para indivíduos (atletas) que cronicamente ingerem este composto. O músculo humano parece ter um limite máximo para armazenar esta substância, 150 - 160 mmol/kg de músculo seco (Guerrero-Ontiveros \& Wallimann, 1998). Isto sugere influência do seu consumo crônico na síntese endógena, objetivando prevenir uma acumulação excessiva de creatina intramuscular. Esta supressão da expressão da proteína transportadora deste composto pode ser interpretada como um efeito colateral da suplementação. Não é aconselhavel o consumo por longo tempo, sendo recomendado o período de 1 mês sem consumo de creatina, após 3 meses de suplementação contínua (Guerrero-Ontiveros \&Wallimann, 1998).

A suplementação com creatina poderia significar uma desvantagem para alguns atletas. O fato de este composto poder aumentar o peso corporal tem sido considerado. Como desvantagem em esportes basicamente aeróbios, isto é, o aumento do peso implicaria em custo energético adicional para movimentar o peso do corredor (Williams, 1998).

Ainda não se apresentam claramente definidos os efeitos colaterais decorrentes da suplementação crônica com creatina. Conseqüentemente, mais pesquisas são necessárias.

\section{O N CLUS Ã O}

O consumo de creatina, substância ergogênica não considerada como doping pelo Comitê Olímpico Internacional, tem se mostrado efetivo na melhoria do desempenho esportivo, porém, em condições específicas de exercício, principalmente em modalidades de curta duração, alta intensidade e períodos curtos de recuperação. Este efeito seria devido ao aumento dos níveis musculares de creatina, o qual poderia potencializar a rápida regeneração do ATP. A suplementação seria mais efetiva naqueles indivíduos com níveis iniciais baixos deste 
composto nos músculos, como vegetarianos e idosos. Os efeitos ergogênicos desta substância podem ser aumentados quando consumida com glicose, mas a quantidade do carboidrato deve ser grande. É importante ressaltar que não são poucos os trabalhos bem controlados sem demonstração de benefício significativo com o consumo de creatina. Não há, ainda, evidência conclusiva sobre efeitos colaterais de seu uso. Faltam também mais estudos para determinar se o aumento de peso decorrente do seu consumo é devido à retenção de água ou a um aumento verdadeiro da síntese de proteínas.

\section{REFERÊ NCIAS BIBLIOGRÁFICAS}

AMERICAN COLLEGE OF SPORTS MEDICINE. The physiological and health effects of oral creatine supplementation. Medicine and Science in Sports and Exercise, Madison, v.32, n.3, p.706-717, 1999.

BALSOM, P., SODERLUND, K., EKBLOM, B. Creatine in humans with special reference to creatine supplementation. Sports Medicine, Auckland, v.18, n.4, p.268-280, 1994.

BALSOM, P., SODERLUND, K., SJODIN, B., EKBLOM, B. Skeletal muscle metabolism during short duration high intensity exercise: influence of creatine supplementation. Acta Physiologica Scandinavica, Stockholm, v.154, n.3, p.303-310, 1995.

BROBERG, S., SAHLIN, K. Adenine nucleotide degradation in human skeletal muscle during prolonged exercise. Journal of Applied Physiology, Bethesda, v.67, n.1, p.116-122, 1989.

BURKE, E., BERNING, J. Nutritional ergogenics aids. In: BURKE, E., BERNING, J. Training nutrition. Carmel : Cooper P.G., 1996. p.94-96.

DAWSON, B., CUTLER, M., MOODY, A., LAWRENCE, S., GOODMAN, C., RANDALL, N. Effect of oral creatine loading on single and repeated maximal shorts sprints. Australian Journal of Science Medicine Sports, Camberra, v.27, n.3, p.56-61, 1995.
DEVLIN, T. Textbook of biochemistry: with clinical correlations. New York: Wiley-Liss, 1992. 518p.

EDSTRÖM, L., HULTMAN, E., SAHLIN, K. The contents of high-energy phosphates in different fiber types in skeletal muscles from rat, guinea pig and man. Journal of Physiology, Masson, v.332, p.47-58, 1982.

FORBES, G. Composición del organismo. In: ORGANIZACIÓN MUNDIAL DE LA SALUD. Conocimientos actuales de nutrición. 6.ed. Washington : [s.n.], 1991. p.57-68. (Publicación Científica, n.532).

GARIOD, L., BINZONI, T., FERETTI, G. Standardization of 31 phosphorus-nuclear magnetic resonance spectroscopy determinations of high-energy phosphates in humans. European Journal of Applied Physiology, Berlin, v.68, n.2, p.107-110, 1994.

GREEN, A.L., SIMPSOM, E.S., LITTLEWOOD, J.S., MacDONALD, I.A., GREENHAFF, P.L. Carbohydrate ingestion augments creatine retention during creatine feeding in humans. Acta Physiologica Scandinavica, Stockholm, v.158, n.2, p.195-202, 1996.

GREENHAFF, P., BODIN, K., HARRIS, R., HULTMAN, E., JONES, D.D., MCINTYRE, D. The influence of oral creatine supplementation on muscle creatine resynthesis following intense contraction in man. Journal of Physiology, Masson, v.467, p.75, 1993. (Abstract).

GREENHAFF, P.L., NEVILL, M.E., SODERLUND, K., BODIN, K., BOOBIS, L.H., WILLIAMS, C., HULTNAN, $E$. The metabolic response of human type I and II muscles fibers during maximal treadmill sprinting. Journal of Physiology, Masson, v.478 (Parte 1), p.149-155, 1994.

GREENHAFF, P.L. Creatine and its application as an ergogenic aid. International Journal of Sports Nutrition, Sttutgart, v.5, p.S100-S110, 1995. Supplement.

GREENHAFF, P. The nutritional biochemistry of creatine. Journal of Nutritional Biochemistry, Stoneham, v.11, p.610-618, 1997.

GRINDSTAFF, P., KREIDER, R., BISHOP, R., WILSON, M., WOOD, L., ALEXANDER, C., ALMADA, A. Effects 
of creatine supplementation on repetitive sprint performance and body composition in competitive swimmers. International Journal of Sports Nutrition, Sttutgart, v.7, n.4, p.330-346, 1997.

GUERRERO-ONTIVEROS, M.L., WALLIMANN, T. Creatine supplementation in health and disease of chronic creatine ingestion in vivo: Down-regulation of the expression of creatine transporter isoforms in skeletal muscles. Molecular and celular Biochemistry, Washington, v.184, n.1/2, p.427-437, 1998.

HARRIS, R.C., SODERLUND, K., HULTMAN, E. Elevation of creatine in resting and exercised muscle of normal subjects by creatine supplementation. Clinical Science, Colchester, v.83, n.3, p.367-374, 1992.

HOUSTON, M. Biochemical energetics. In: HOUSTON, M. Biochemistry primer for exercise science. Champaign: Human Kinetics, 1995. p.49-56.

HULTMAN, E., SODERLUND, K., TIMMONS, J.A., CEDERBLAD, G., GREENHAFF, P.L. Muscle creatine loading in men. Journal of Applied Physiology, Bethesda, v.81, n.1, p.232-237, 1996.

KREIDER, R. Creatine, the next ergogenic supplement? Disponível em: <URL:http://www.sportsci.org/ traintech/creatine/rbk.html>. Acesso em: 1998.

MCCULLY, K., POSNER, J. Measuring exercise-induced adaptations and injury with magnetic resonance spectroscopy. International Journal of Sports Medicine, Sttutgart, v.13, p.S147-S149, 1992. Supplement 1.

MAYES, P. The respiration chain and oxidative phosphorylation. In: MURRAY, R., GRANNER, D., MAYES, P., RODWELL, V. (Ed.). Harper's biochemistry. 24.ed. Stamford: Lange, 1996. p.123-134.

MOLLER, P., BERGSTROM, J., FURST, P. Effect of aging on energy rich phosphagens in human skeletal muscles. Clinical Science, Colchester, v.58, n.6, p.553-555, 1980.

MOLLER, P., BRANDT, R. Skeletal muscle adaptation to aging and to respiratory and liver failure. Stockholm, 1981. (Dissertation) - Karolinska Institute, 1981.

NEWSHOLME, E., LEECH, T., DEUSTER, G. Keep on running. Chichester : John Wiley \& Sons., 1994. p.50-69.
ODOOM, J.E., KEMP, G.J., RADDA, G.K. The regulation of total creatine content in amyoblast cell line. Molecular and Celular Biochemistry, Washington DC, v.158, n.2, p.179-188, 1996.

POORTMANS, J.R., AUQUIER, H., RENAUT, V., DURASSEL, A., SAUGY, M., BRISSON, G.R. Effect of short-term creatine supplementation on renal responses in men. European Journal of Applied Physiology, Berlin, v.76, n.6, p.566-567, 1997.

REDONDO, D.R., DOWLING, E.A., GRAHAM, B.L., ALMADA, A.L., WILLIAMS, M.H. The effect of oral creatine monohydrate supplementation on running velocity. International Journal of Sports Nutrition, Sttutgart, v.6, n.3, p.213-221, 1996.

RODWELL, V. Conversion of aminoacids to specialized products. In: MURRAY, R., GRANNER, D., MAYES, P., RODWELL, V. (Ed.). Harper's biochemistry. 24.ed. Stamford : Lange, 1996. p.341-362.

SMITH, S., MONTAIN, S., MATOTT, R., ZIENTARA, G., JOLESZ, F., FIELDING, R. Creatine supplementation and age influence muscle metabolism during exercise. Journal of Applied Physiology, Bethesda, v.85, n.4, p.1349-1356, 1998.

SODERLUND, K., HULTMAN, E. ATP and phosphocreatine changes in single human muscle fibers after intense electrical stimulation. American Journal of Physiology, Bethesda, v.261, n.6 (Part 1), p.E737-E741, 1991.

SODERLUND, K., GREENHAFF, P.L., HULTMAN, E. Energy metabolism in type I and II human muscle fibres during short term electrical stimulation at different frequencies. Acta Physiologica Scandinavica, Stockholm, v.144, n.1, p.15-22, 1992.

SODERLUND, K., BALSOM, P., EKBLOM, B. Creatine supplementation and high intensity exercise: influence on performance and muscle metabolism. Clinical Science, Colchester, v. 87 p.120-121, 1994. Supplement.

SPRIET, L. Anaerobic metabolism during high-intensity exercise. In: HARGREAVES, M. (Ed.). Exercise metabolism. Champaign : Human Kinetics, 1995. p.1-39. 
TERRILLION, K.A., KOLKHORST, F.W., DOLGENER, F.A., JOSLYN, S.J. The effect of creatine supplementation on two 700-m maximal running bouts. International Journal of Sports Nutrition, Sttutgart, v.7, n.2, p.138-143, 1997.

THOMPSON, C.H., KEMP, G.J., SANDERSON, A.L., RADDA, G.K. Skeletal muscle mithocondrial function studied by kinetic analysis of postexercise creatine resynthesis. Journal of Applied Physiology, Bethesda, v.78, p.2131-2139, 1995.

VANDENBERGHE, K., GILLIS, N., Van HECKE, P., Van LEEMPUTTE, M., VANSTAPEL, F., HESPEL, P. Caffeine counteracts the ergogenic action of muscle creatine loading. Journal of Applied Physiology, Bethesda, v.80, n.2, p.452-457, 1996.

VANDENBERGHE, K., GILLIS, N., Van HECKE, P., Van LEEMPUTTE, M., VANGERVEN, L., HESPEL, P. Long term creatine intake is beneficial to muscle performance during resistance training. Journal of Applied Physiology, Bethesda, v.83, n.6, p.2055-2063, 1997.

VOLEK, J.S., KRAEMER, W.J., BUSH, J.A., BOETES, M., INCLEDON, T., CLARK, K.L., LYNCH, J.M. Creatine supplementation enhance muscular performance during high intensity resistance exercise. Journal of American Dietetic Association, Chicago, v.97, n.7, p.765-770, 1997.

WALKER, J. Creatine biosynthesis, regulation and function. Advances in Enzymology and Related Areas in Mollecular Biology, v.50, p.117-142, 1979.

WILLIAMS, M. Rating the sports ergogenics. The ergogenic edge. Champaing : Human Kinetics, 1998. p.178-182.

WILMORE, J., COSTILL, D. Basic energy systems. In: WILMORE, J., COSTILL, D. (Ed.). Physiology of sport and exercise. Champaign: Human Kinetics, 1994. p.92-121.

ZIEGENFUSS, T., LEMON, P., ROGERS, M., ROSS, R., YARASHESKI, K. Acute creatine ingestion: effects on muscle volume, anaerobic power, fluid volumes, and protein turnover. Medicine Science in Sports and Exercise, Madison, v.29 p.S127, 1997. (Abstract).

Recebido para publicação em 13 de dezembro de 2000 e aceito em 1 de junho de 2001. 